\title{
Coordination games with vanishing actions
}

Citation for published version (APA):

Schoenmakers, G., Flesch, J., \& Thuijsman, F. (2002). Coordination games with vanishing actions. International Game Theory Review, 04(02), 119-126. https://doi.org/10.1142/S0219198902000598

Document status and date:

Published: 01/01/2002

DOI:

10.1142/S0219198902000598

Document Version:

Publisher's PDF, also known as Version of record

Document license:

Taverne

\section{Please check the document version of this publication:}

- A submitted manuscript is the version of the article upon submission and before peer-review. There can be important differences between the submitted version and the official published version of record.

People interested in the research are advised to contact the author for the final version of the publication, or visit the DOI to the publisher's website.

- The final author version and the galley proof are versions of the publication after peer review.

- The final published version features the final layout of the paper including the volume, issue and page numbers.

Link to publication

\footnotetext{
General rights rights.

- You may freely distribute the URL identifying the publication in the public portal. please follow below link for the End User Agreement:

www.umlib.nl/taverne-license

Take down policy

If you believe that this document breaches copyright please contact us at:

repository@maastrichtuniversity.nl

providing details and we will investigate your claim.
}

Copyright and moral rights for the publications made accessible in the public portal are retained by the authors and/or other copyright owners and it is a condition of accessing publications that users recognise and abide by the legal requirements associated with these

- Users may download and print one copy of any publication from the public portal for the purpose of private study or research.

- You may not further distribute the material or use it for any profit-making activity or commercial gain

If the publication is distributed under the terms of Article $25 \mathrm{fa}$ of the Dutch Copyright Act, indicated by the "Taverne" license above, 
International Game Theory Review, Vol. 4, No. 2 (2002) 119-126

(C) World Scientific Publishing Company

\title{
COORDINATION GAMES WITH VANISHING ACTIONS
}

\author{
G. SCHOENMAKERS, * J. FLESCH and F. THUIJSMAN \\ Department of Mathematics, Maastricht University, \\ P.O. Box 616, 6200 MD Maastricht, The Netherlands \\ *gm.schoenmakers@math.unimaas.nl
}

\begin{abstract}
We deal with $n$-player coordination games with vanishing actions, which are repeated games where all non-diagonal entries yield zero-payoffs, and where, moreover, at any stage beyond $r_{i}$ any player $i$ loses any action that she has not used during the previous $r_{i}$ stages of play. For these games we examine the set of equilibrium rewards, where we treat the two-player case and the more player case separately. Folk-theorem like results are established.
\end{abstract}

Keywords: Repeated games; equilibrium; Folk theorem.

\section{Introduction}

An $n$-player coordination game is a repeated game where all players have the same number of actions, and where all non-diagonal payoffs are 0 . Moreover we shall assume that all diagonal payoffs are strictly positive. Since these games are just a special type of repeated games, the Folk-theorem applies, i.e., the set of equilibrium rewards is the set of all feasible and individually rational rewards [cf. e.g., Aumann (1981) or Sorin (1992)]. Here a player's reward is to be interpreted as the limiting average of her stage payoffs.

In this paper we generalize upon the idea of vanishing actions introduced by Joosten, Peters and Thuijsman (1995) for zero-sum repeated games. The idea behind vanishing actions is forgetfulness or unlearning of those actions that have not been used for a specified number of stages. One can think of a surgeon who unlearns certain surgical skills when not exercised at some, more or less, regular basis. More precisely, a number $r$ is specified which is to be interpreted as follows: at any stage beyond $r$, a player's action $a$ will vanish from her action set, when this player has not played $a$ at any of the previous $r$ stages. Actions that are vanished are no longer available in the remainder of the play. Thus, at any stage of play, players have to balance their interest between achieving a high payoff and keeping the right actions available. Thus we get an $r$-restricted coordination game.

This type of games can also be interpreted as a special class of stochastic games with finite state and action spaces. Such games were introduced by Shapley (1953),

${ }^{*}$ Correspondence author. 
but for the general $n$-player case the existence of equilibria is still unknown, although for certain specially structured classes they have been shown to exist. A survey of the state of the art can be found in Neyman and Sorin (2001).

In Sec. 2 we will present the model in more detail and examine a first example, where $r \leq 2$. Next we focus on games with $r \geq 3$. In Sec. 3 we show that for two-player games of this type any convex combination of the diagonal payoffs can be obtained as an equilibrium reward. We also show that a Folk-theorem like result does not apply for this class of games, since there may be feasible and individually rational rewards outside this diagonal hull which cannot be achieved as equilibrium rewards. In Sec. 4 an extension to multiplayer coordination games is made and it is exhibited that all feasible rewards can be obtained as equilibrium rewards. In Sec. 5 we extend the results to the case where each player $i$ is allowed to have her own level of unlearning $r^{i}$.

\section{The Model}

An $n$-player $r$-restricted coordination game is determined by the following parameters:

(1) $I=\{1,2, \ldots, n\}$ is the set of players, with $n \geq 2$;

(2) $A^{i}=\{1,2, \ldots, m\}$ is the initial set of pure actions for player $i$, with $m \geq 2$;

(3) $u^{i}: \times_{i=1}^{n} A^{i} \rightarrow \mathbb{R}$ is the payoff function for player $i$; we assume that $u^{i}\left(a^{1}\right.$, $\left.a^{2}, \ldots, a^{n}\right)>0$ if $a^{1}=a^{2}=\cdots=a^{n}$ and $u^{i}\left(a^{1}, a^{2}, \ldots, a^{n}\right)=0$ otherwise;

(4) $r$ is the level of unlearning for the players.

The game is played at stages $1,2, \ldots$, where each time simultaneously and independently each player $i$ has to choose one of her available actions $a^{i}$. The action choices are announced and each player $i$ receives a payoff $u^{i}\left(a^{1}, a^{2}, \ldots, a^{n}\right)$. Then play moves to the next stage, where actions have to be chosen again. However, actions may disappear according to the following rule: At any stage beyond $r$, a player's action $a$ will vanish from her action set, when this player has not played $a$ at any of the previous $r$ stages. Thus the number of available actions may decrease during the course of play. Therefore we shall use $A_{t}^{i}$ to denote the set of feasible or not (yet) unlearned pure actions of player $i$ at stage $t$ and we define $h_{t}$ to be the history of play up to stage $t$. At any stage $t$ any player $i$ is allowed to randomize over the available actions in $A_{t}^{i}$, which yields a so called mixed action denoted $x_{t}^{i}$ for player $i$ at stage $t$, and this choice obviously may depend on $h_{t}$. Each pure action is a mixed action as well. A sequence $x^{i}=\left(x_{t}^{i}\right)_{t=1}^{\infty}$ is called a strategy for player $i$. Each player $i$ is assumed to maximize her limiting average reward, i.e.,

$$
\gamma^{i}\left(x^{1}, x^{2}, \ldots, x^{n}\right)=\liminf _{T \rightarrow \infty} \frac{1}{T} \sum_{t=1}^{T} E\left(U_{t}^{i}\right),
$$

where $E\left(U_{t}^{i}\right)$ denotes the expected payoff to player $i$ at stage $t$ given that $\left(x^{1}, x^{2}, \ldots, x^{n}\right)$ is being played. A joint strategy $\left(x^{1}, x^{2}, \ldots, x^{n}\right)$ is an equilibrium if 
for each player $i$ it holds that $\gamma^{i}\left(x^{1}, \ldots, x^{\prime}, \ldots, x^{n}\right) \leq \gamma^{i}\left(x^{1}, \ldots, x^{i}, \ldots, x^{n}\right)$ for all $x^{\prime}$, i.e., each player is playing a best reply against the strategies of the others.

We would like to remark that a 1-restriction implies that the game is essentially a one-shot game, and therefore, every equilibrium in the one-shot game is an equilibrium in the 1-restricted game and vice versa. The following example discusses the 2-restricted case.

Example 2.1. Consider the following 2-player 2-restricted coordination game:

$$
\begin{array}{cc}
L & R \\
T & \left(\begin{array}{ll}
2,1 & 0,0 \\
0,0 & 1,3
\end{array}\right) .
\end{array}
$$

Player 1 chooses rows, player 2 chooses columns. Let $E^{*}$ denote the set of equilibrium rewards for this game. Clearly $(2,1),(1,3) \in E^{*}$, since playing $(T, L)$ forever is an equilibrium that yields $(2,1)$ and playing $(B, R)$ is an equilibrium that gives $(1,3)$. Given the 2-restriction, the only equilibrium where none of the actions vanishes, is the one where $(T, L)$ and $(B, R)$ are played in turns resulting in a $\left(\frac{3}{2}, 2\right)$ equilibrium reward. Since, with certainty, each player has both actions available after stage 1 , we can use the first stage to decide which of these equilibria to play for the remaining stages. One could decide, for example, to play at stage 1 a mixed action according to an equilibrium in the one-shot game $\left(\begin{array}{ll}2,1 & 1,3 \\ 1,3 & \frac{3}{2}, 2\end{array}\right)$ and to continue on any equilibrium in the coordination game for the entry selected. There are still some other ways of generating equilibrium rewards, but we do not have the intention to characterize the set $E^{*}$ at this point. We note, however, that not all rewards in the diagonal hull, i.e., the convex hull of the diagonal payoffs, can be obtained. Take for example $(1.9,1.2)=0.9 \cdot(2,1)+0.1 \cdot(1,3)$. This can be seen by observing that, if we wish to obtain a diagonal reward, then the players can randomize their action at most once, for otherwise a $(0,0)$ reward results with positive probability which implies an off-diagonal average reward. Also, this randomization should be done at stage 1 . There are only finitely many rewards that correspond to pure equilibria and after stage 1 play will evolve to one of those. Therefore there are essentially only finitely many ways to play an equilibrium right from the beginning.

We will show that for $r \geq 3$ the situation is very much different and we shall concentrate on that case from now on. We examine one more example to introduce some concepts.

Example 2.2. Consider the following 2-player 3-restricted game.

$$
\left(\begin{array}{lll}
4,1 & 0,0 & 0,0 \\
0,0 & 2,2 & 0,0 \\
0,0 & 0,0 & 1,4
\end{array}\right) .
$$

The diagonal hull is the triangle with extreme points $(4,1),(2,2)$ and $(1,4)$. Clearly, each diagonal entry corresponds to a pure equilibrium. We shall use the name point 
agreement to address such equilibria. Any reward on a line segment between two diagonal payoffs can also be obtained as an equilibrium reward. We shall use the name line agreement to address equilibria of this type. Take for example $\left(\frac{26}{9}, \frac{14}{9}\right)=$ $\frac{4}{9} \cdot(4,1)+\frac{5}{9} \cdot(2,2)$. A line agreement that yields this reward is for example the one where each player plays the sequence of actions:

$$
1,2,1,1,2,2,1,2,2
$$

repeatedly. More generally, one can take any series

$$
1,2, a_{1}, 1,2, a_{2}, 1,2, a_{3}, 1,2, a_{4}, 1,2, a_{5}, \ldots,
$$

where $a_{k} \in\{1,2\}$ for all $k$ can be chosen arbitrarily. In doing so an equilibrium reward $\lambda \cdot(4,1)+(1-\lambda) \cdot(2,2)$ can be obtained for any $\lambda \in\left[\frac{1}{3}, \frac{2}{3}\right]$. Convex combinations for other $\lambda$ can be obtained as equilibrium rewards by randomization as will be explained in the next section. Using randomization we can also achieve convex combinations of more than two payoffs as equilibrium rewards.

\section{2-Player 3-Restricted Games}

In this section we show that in the 2-player case every convex combination of diagonal payoffs can be obtained as an equilibrium reward. We proceed in two steps: first we show it for convex combinations of only two diagonal payoffs by means of a line agreement, next we extend the result to the general case.

Lemma 3.1. Consider a 2-player 3-restricted coordination game of the form

$$
\left(\begin{array}{cc}
y_{1}, z_{1} & 0,0 \\
0,0 & y_{2}, z_{2}
\end{array}\right)
$$

(with $\left.y_{1}, y_{2}, z_{1}, z_{2}>0\right)$. Then every convex combination of $\left(y_{1}, z_{1}\right)$ and $\left(y_{2}, z_{2}\right)$ can be obtained as an equilibrium reward.

Proof. For each $\lambda \in[0,1]$ define $f(\lambda):=\lambda\left(y_{1}, z_{1}\right)+(1-\lambda)\left(y_{2}, z_{2}\right)$. In four steps we will show that $f(\lambda)$ is an equilibrium reward for each $\lambda \in[0,1]$.

Step 1: $f(1)=\left(y_{1}, z_{1}\right)$ and $f(0)=\left(y_{2}, z_{2}\right)$ can be obtained as equilibrium rewards. Take $x_{1}^{1}=x_{1}^{2}=(1,0)^{\infty}$ and $x_{0}^{1}=x_{0}^{2}=(0,1)^{\infty}$. Then $\gamma\left(x_{1}^{1}, x_{1}^{2}\right)=f(1)=$ $\left(y_{1}, z_{1}\right)$ and $\gamma\left(x_{0}^{1}, x_{0}^{2}\right)=f(0)=\left(y_{2}, z_{2}\right)$ and both pairs are equilibria.

Step 2: For any $\lambda \in\left[\frac{1}{3}, \frac{2}{3}\right]$ the reward $f(\lambda)$ can be obtained by a line agreement. Take $\lambda \in\left[\frac{1}{3}, \frac{2}{3}\right]$. Define the pure strategies $x_{\lambda}^{1}=x_{\lambda}^{2}=\left(1,2, a_{1}, 1,2, a_{2}\right.$, $\left.1,2, a_{3}, \ldots\right)$ where for all $i$ the actions $a_{i} \in\{1,2\}$ are such that the long run frequency of action 1 is $\lambda$. Then $\gamma\left(x_{\lambda}^{1}, x_{\lambda}^{2}\right)=\lambda\left(y_{1}, z_{1}\right)+(1-\lambda)\left(y_{2}, z_{2}\right)=f(\lambda)$ and obviously neither player can make a profitable unilateral deviation.

Step 3: For any $\lambda \in\left[\frac{1}{6}, \frac{1}{3}\right] \cup\left[\frac{2}{3}, \frac{5}{6}\right]$ the reward $f(\lambda)$ can be obtained by a line agreement. Without loss of generality we take $\lambda \in\left[\frac{1}{6}, \frac{1}{3}\right]$. Note that $f(\lambda)=$ $\frac{1}{2} f(0)+\frac{1}{2} f(2 \lambda)$. From step 1 we know that $\left(x_{0}^{1}, x_{0}^{2}\right)$ leads to a reward of $f(0)$ 
and from step 2 we know the existence of a line agreement $\left(x_{2 \lambda}^{1}, x_{2 \lambda}^{2}\right)$ with reward $f(2 \lambda)$. We will now define strategies that result in playing either $\left(x_{0}^{1}, x_{0}^{2}\right)$ or $\left(x_{2 \lambda}^{1}, x_{2 \lambda}^{2}\right)$, each with probability $\frac{1}{2}$ from stage 2 onwards: Define $\left(L_{\lambda}^{1}, L_{\lambda}^{2}\right)$ by playing $\left(\left(\frac{1}{2}, \frac{1}{2}\right),\left(\frac{1}{2}, \frac{1}{2}\right)\right)$ at stage 1 , followed by playing $\left(x_{0}^{1}, x_{0}^{2}\right)$ if the first stage actions are the same, and followed by $\left(x_{2 \lambda}^{1}, x_{2 \lambda}^{2}\right)$ otherwise. Then $\left(L_{\lambda}^{1}, L_{\lambda}^{2}\right)$ is an equilibrium as required.

Step 4: For any $\lambda \in[0,1]$ the reward $f(\lambda)$ can be obtained by a line agreement. Take $\lambda \in\left[\frac{1}{12}, \frac{1}{6}\right]$ and suppose that $\left(L_{2 \lambda}^{1}, L_{2 \lambda}^{2}\right)$ is an equilibrium as in the proof of step 3 . We will now introduce strategies that result in playing either $\left(x_{0}^{1}, x_{0}^{2}\right)$ or $\left(L_{2 \lambda}^{1}, L_{2 \lambda}^{2}\right)$, each with probability $\frac{1}{2}$ from stage 3 onwards: Define $\left(L_{\lambda}^{1}, L_{\lambda}^{2}\right)$ by playing $\left(\frac{1}{2}, \frac{1}{2}\right)$ at stage 1 , followed by playing $\left(x_{0}^{1}, x_{0}^{2}\right)$ if the first stage actions are the same, and followed by the alternative action at stage 2 and from stage 3 onwards start playing $\left(L_{2 \lambda}^{1}, L_{2 \lambda}^{2}\right)$, otherwise. When the latter is done stage 3 acts as if it were the initial stage. Then $\left(L_{\lambda}^{1}, L_{\lambda}^{2}\right)$ is an equilibrium as required. The alternative actions at stage 2 are necessary in order not to lose any action in the selection process. Thus we have established equilibria for all rewards $f(\lambda)$ with $\lambda \in\left[\frac{1}{12}, \frac{1}{6}\right]$. By repeating this very same procedure as often as we like we obtain the statement of the lemma.

Theorem 3.1. Consider a 2-player 3-restricted coordination game. Then every convex combination of the diagonal payoffs can be obtained as an equilibrium reward.

Proof. If $\left(u^{1}, u^{2}\right)$ is in the diagonal hull, then $\left(u^{1}, u^{2}\right)$ is a convex combination of at most three diagonal payoffs $D_{i}, D_{j}$ and $D_{k}$ (because any two-dimensional polytope can be subdivided in triangles with the same set of extreme points). Without loss of generality, suppose that $\left(u^{1}, u^{2}\right)=\alpha_{1} \cdot D_{i}+\alpha_{2} \cdot D_{j}+\alpha_{3} \cdot D_{k}$, where $\alpha=\left(\alpha_{1}, \alpha_{2}, \alpha_{3}\right) \in$ $\Delta^{3}$, the unit simplex in $\mathbb{R}^{3}$, and suppose that $\alpha_{1} \geq \alpha_{2} \geq \alpha_{3}$.

Let $\mu=\left(\frac{3}{2}\left(\alpha_{1}+\alpha_{3}\right)-\frac{1}{2}, \frac{3}{2} \alpha_{2}, 0\right) \in \Delta^{3}$ and $\xi=\left(1-3 \alpha_{3}, 0,3 \alpha_{3}\right) \in \Delta^{3}$, and write $D=\left(D_{i}, D_{j}, D_{k}\right)$. Then

$$
\left(u^{1}, u^{2}\right)=\left(\frac{2}{3} \mu+\frac{1}{3} \xi\right) \cdot D .
$$

Since both $\mu$ and $\xi$ only put positive weight on at most two diagonal payoffs, we can use line agreements $L_{\mu}$ and $L_{\xi}$ to support $\mu \cdot D$ and $\xi \cdot D$ as equilibrium rewards respectively. We will now define strategies that result in playing either $L_{\mu}$ or $L_{\xi}$ with probability $\frac{2}{3}$ and $\frac{1}{3}$ respectively, from stage 2 onwards. We can do so by having the players play actions $i, j$ and $k$ each with probability $\frac{1}{3}$ at stage 1 , followed by playing $L_{\xi}$ if the first stage actions are the same, and followed by $L_{\mu}$ otherwise.

We close this section by an example which shows that in the 2-player case of restricted coordination games it may be possible to have feasible individually rational rewards that cannot be obtained as equilibrium rewards. 
Example 3.1. Consider the following 3-restricted coordination game:

$$
\begin{aligned}
& L \quad R \\
& \text { B }\left(\begin{array}{ll}
2,1 & 0,0 \\
0,0 & 1,1
\end{array}\right) \text {. }
\end{aligned}
$$

From Joosten, Peters and Thuijsman (1995) it can be derived that for this game any payoff greater or equal to $\left(\frac{3}{4}, \frac{1}{2}\right)$ is individually rational. Thus $\left(\frac{3}{4}, \frac{3}{4}\right)$ is a feasible and individually rational payoff. However, it can be seen that this payoff cannot be obtained as an equilibrium reward using the following arguments. Suppose, by way of contradiction that we have an equilibrium for this reward. Then the long run frequency of diagonal payoff $(2,1)$ should be 0 , because one can only obtain $\left(\frac{3}{4}, \frac{3}{4}\right)$ as a convex combination of the form $\frac{3}{4}(1,1)+\frac{1}{4}(0,0)$. Thus, we distinguish three cases: (1) If both players keep both actions available throughout play, then the frequency of the payoff $(0,0)$ should be at least $\frac{2}{3}$. But then, player 1 receives less than $\frac{3}{4}$ although he could always achieve $\frac{3}{4}$. Therefore this contradicts the equilibrium condition. (2) If at any stage exactly one player has both actions available, then the $(0,0)$ payoff should have a frequency of 0 , since this player would go for the positive payoff. (3) If both players keep only one action, then the remaining entry can not be a $(0,0)$ entry for the following reason. Suppose that entry $(B, L)$ would remain, then this can only happen if at some stage $(B, L)$ has been played twice consecutively, while all actions are still available (for otherwise we would be in case $(2)$ ). If at this stage entry $(T, R)$ is played with positive probability, then player 2 will loose action $R$ with positive probability and player 1 could continue by playing $T$ for the remaining play. However, then payoff $(2,1)$ has a positive frequency, which is a contradiction. If player 2 plays action $R$ with probability 1 , then we do not get absorption in entry $(B, L)$. Finally, if player 1 plays action $B$ with probability 1 , then player 2 would play $R$ with probability 1 . Summarizing, payoff $(0,0)$ always ends up with frequency 0 . This completes the argument.

\section{Multiplayer 3-Restricted Games}

Although not true for the 2-player case (cf. Example 3.1), we now show that for the $n$-player case with $n \geq 3$ the set of equilibrium rewards in 3 -restricted coordination games equals the set of feasible individually rational rewards. The proof uses Lemmas 4.1 and 4.2 below.

Lemma 4.1. Let $k \geq 2$. For any $z \in \Delta^{k}$ there exist $y_{1}, y_{2}, \ldots, y_{k-1} \in \Delta^{k}$ such that $z=\frac{1}{k-1} \sum_{i=1}^{k-1} y_{i}$ and for any $y_{i}$ at most two coordinates are nonzero.

Proof. We prove the result by induction. If $k=2$, then $z$ has at most two nonzero coordinates, so we can take $y_{1}=z$. Suppose the result is true for $k$. We now show that it is also true for $k+1$. Take $z=\left(z_{1}, z_{2}, \ldots, z_{k+1}\right) \in \Delta^{k+1}$ and suppose, without loss of generality, that $z_{1} \geq z_{2} \geq \cdots \geq z_{k} \geq z_{k+1}$. Notice that $k z_{1}+z_{k+1} \geq$ 
$z_{1}+z_{2}+\cdots z_{k+1}=1$, so $z_{1}-\frac{1}{k}+z_{k+1} \geq 0$. Then by letting $w:=\left(\frac{k}{k-1}\left(z_{1}-\frac{1}{k}+\right.\right.$ $\left.\left.z_{k+1}, z_{2}, \ldots, z_{k}\right)\right) \in \Delta^{k}$ and $y_{k}:=\left(1-k z_{k+1}, 0, \ldots, 0, k z_{k+1}\right) \in \Delta^{k+1}$ we have

$$
z=\left(z_{1}, z_{2}, \ldots, z_{k}, z_{k+1}\right)=\frac{k-1}{k} \cdot(w, 0)+\frac{1}{k} \cdot y_{k} .
$$

By induction there are $w_{1}, w_{2}, \ldots, w_{k-1} \in \Delta^{k}$, such that $w=\frac{1}{k-1} \sum_{l=1}^{k-1} w_{l}$, while each $w_{l}$ has at most two nonzero coordinates. Then, by letting $y_{l}=\left(w_{l}, 0\right)$ for $l=1,2, \ldots, k-1$, we get

$$
z=\frac{1}{k} \sum_{l=1}^{k} y_{l}
$$

which completes the proof.

Lemma 4.2. Let $n \geq 3$. In any $n$-player 3 -restricted coordination game $(0,0, \ldots, 0)$ can be obtained as an equilibrium reward.

Proof. Recall that each player has at least 2 actions. Let player 1 play $(1,2,1,2, \ldots)$ and let player 2 play $(2,1,2,1, \ldots)$, and let all other players play $(1,1,1,1, \ldots)$, while in addition if player 2 does not play according to this plan, then player 1 continues by playing action 2 exclusively and similarly, if player 1 does not play according to this plan, then player 2 continues by playing action 2 exclusively. Then, clearly, the rewards are 0 and none of the players has a profitable deviation.

Theorem 4.1. Let $n \geq 3$. In any $n$-player 3 -restricted coordination game all points inside the payoff hull can be obtained as limiting average equilibrium rewards.

Proof. The proof proceeds in a number of steps. In step 1 we prove that any convex combination of two payoffs can be obtained as an equilibrium reward by a line agreement. In step 2 we fill the space between the lines.

Step 1: Any convex combination of two diagonal payoffs can be obtained in a similar way as this was done for the 2-player case in Lemma 3.1. Now take a convex combination of 0 and a diagonal payoff, let's say $D_{1}$ corresponding to action 1 for all players. So we can write this convex combination as $\lambda D_{1}$. Then, similarly to the 2-player case we can get $\lambda D_{1}$ by focussing on entries $(1,1, \ldots, 1)$ giving $D_{1}$ and $(2,2,1,1, \ldots, 1)$ giving 0 . Almost the same strategies can be used as in the 2-player case; the only difference is that in case $n=3$ players 1 and 2 should prevent player 3 from playing action 2 along with them; this can be done by using the threat that players 1 and 2 will play actions 1 and 2 respectively from that moment onwards. As far as the randomizations are concerned, it is only players 1 and 2 who may need to randomize and they only randomize on actions 1 and 2 . Thus every "linepayoff", i.e., every convex combination of two payoffs, can be obtained by a line agreement. 
Step 2: If all players have $m$ actions then there are at most $m+1$ different payoffs. Let $u$ be an arbitrary convex combination of these $m+1$ payoffs $D_{0}, D_{1}, \ldots, D_{m}$, where $D_{0}=0 \in \mathbb{R}^{n}$. So $u=\sum_{l=0}^{m} \alpha_{l} D_{l}$, where $\alpha \in \Delta^{m+1}$. By Lemma 4.1 there are $\beta_{1}, \beta_{2}, \ldots, \beta_{m} \in \Delta^{m+1}$ such that each $\beta_{j}$ has at most 2 nonzero coordinates and $\alpha=\frac{1}{m} \sum_{j=1}^{m} \beta_{j}$. Then, each $\beta_{j}$ corresponds to a line agreement $L_{j}$, since for each of them at most 2 payoffs are involved. Moreover

$$
u=\sum_{l=0}^{m} \alpha_{l} D_{l}=\frac{1}{m} \sum_{j=1}^{m} \sum_{l=0}^{m} \beta_{j}(l) D_{l}=\frac{1}{m} \sum_{j=1}^{m} \gamma\left(L_{j}\right)
$$

where $\gamma\left(L_{j}\right)$ is the reward corresponding to $L_{j}$. At stage 1 suppose players 1 and 2 both play $\left(\frac{1}{m}, \frac{1}{m}, \ldots, \frac{1}{m}\right)$, while all other players play action 1 . If $(a, b, 1,1, \ldots, 1)$ is the entry selected at stage 1 , then from stage 2 onwards the players play the line agreement that corresponds to $L_{j}$, where $j=$ $(a+b) \bmod m+1$. This yields an equilibrium with reward $u$ which completes the proof.

\section{Concluding Remarks}

Although in the previous sections we have discussed the restricted coordination games in a symmetric setting, i.e., all players having $r=3$ as level of unlearning, all results can be generalized. More specifically, if each player $i$ has her own level of unlearning $r_{i}$, then we obtain the following results.

Theorem 5.1. Consider a 2-player $\left(r_{1}, r_{2}\right)$-restricted coordination game with $r_{1}, r_{2} \geq 3$. Then every convex combination of the diagonal payoffs can be obtained as an equilibrium reward.

Theorem 5.2. Let $n \geq 3$. In any $n$-player $\left(r_{1}, r_{2}, \ldots, r_{n}\right)$-restricted coordination game, with $r_{1}, r_{2}, \ldots, r_{n} \geq 3$, all points inside the payoff hull can be obtained as limiting average equilibrium rewards.

\section{References}

Aumann, R. J. (1981). "Survey of repeated games," Essays in Game Theory and Mathematical Economics in Honor of Oskar Morgenstern (Bibliographisches Institüt, Mannheim, pp. 11-42).

Joosten, R., H. Peters and F. Thuijsman (1995). "Unlearning by not doing: Repeated games with vanishing actions," Games and Economic Behavior 9 pp. 1-7.

Neyman, A. and S. Sorin (2001). Stochastic Games and Applications. NATO ASI Series, Kluwer Academic Publishers, Dordrecht (forthcoming).

Shapley, L. S. (1953). Stochastic games. Proceedings of te National Academy of Sciences U.S.A. 39 pp. $1095-1100$.

Sorin, S. (1992). Repeated Games with Complete Information." In: R. J. Aumann and S. Hart (eds.), Handbook of Game Theory With Economic Applications, Volume 1. Elsevier Science Publishers, North-Holland, pp. 72-107. 